Check for updates

Cite this: RSC Adv., 2017, 7, 19189

Received 12th January 2017

Accepted 15th March 2017

DOI: $10.1039 / \mathrm{c} 7 \mathrm{ra00478h}$

rsc.li/rsc-advances

\title{
Plasma-deposited hydrogenated amorphous silicon films: multiscale modelling reveals key processes $\uparrow$
}

\author{
Z. Marvi, ${ }^{\text {ab }}$ S. Xu, ${ }^{\star a}$ G. Foroutan, ${ }^{\text {b }}$ K. Ostrikov ${ }^{c d}$ and I. Levchenko ${ }^{a d}$
}

The underlying physical and chemical mechanisms and role of the plasma species in the synthesis of hydrogenated amorphous silicon (a-Si:H) thin films were studied numerically with the aim to reveal the key growth processes and, hence, to ensure a much higher level of control over the film structure and properties. A sophisticated multiscale model developed on the basis of self-consistent surface and plasma kinetics sub-models, including one-dimensional plasma sheath formalization, was used to study the nucleation, growth, and structure formation of amorphous hydrogenated silicon films in a reactive low temperature plasma environment containing a mixture of silane, hydrogen, and argon gases. The model considers a whole range of key processes, and the effect of surface temperature, ion flux, energy and other plasma-sheath parameters are examined in detail. The leading role of hydrogen in structure formation is confirmed and moreover, key processes critically important for designing and discovering novel materials with important properties are identified. The dominant role of $\mathrm{SiH}_{3}$ as the main precursor in the deposition of amorphous hydrogenated silicon films is proved, and routes for the efficient, technique-enabled control are specified. The presented results were compared with the experimental data, and a good agreement with the experimental findings obtained for the deposition of amorphous hydrogenated films has been demonstrated.

\section{Introduction}

Hydrogenated amorphous silicon (a-Si:H) films possess many unique properties such as very low light-induced degradation and long wavelength absorption, which provoke a wide application field for this material ${ }^{\mathbf{1 , 2}}$ for the formation of active layers in, e.g., novel highly efficient thin-film based solar cells, ${ }^{3,4}$ thin film and flexible transistors, ${ }^{5,6}$ flat panel displays ${ }^{7,8}$ and other up-to-date nanomaterial-based devices ${ }^{9}$ including flexible and wearable gadgets. Specifically, current research and development efforts on the application of the hydrogenated Si films aim primarily towards designing highly efficient multi-junction photovoltaic devices such as high-voltage solar cells. With an optical bandgap of about $1.6 \mathrm{eV}$ and absorption coefficient of about $4 \times 10^{4} \mathrm{~cm}^{-1}$, this material remains extremely promising. ${ }^{10}$ Another important application of silicon layers is as the

${ }^{a}$ Plasma Sources and Applications Centre, NIE, Nanyang Technological University, 637616 Singapore. E-mail: shuyan.xu@nie.edu.sg

${ }^{b}$ Physics Department, Faculty of Science, Sahand University of Technology, 513351996, Tabriz, Iran

${ }^{c}$ CSIRO-QUT Joint Sustainable Materials and Devices Laboratory, CSIRO, P. O. Box 218, Lindfield, NSW 2070, Australia

${ }^{d}$ School of Chemistry, Physics and Mechanical Engineering, Queensland University of Technology, Brisbane, QLD, Australia

$\dagger$ Electronic supplementary information (ESI) available. See DOI: 10.1039/c7ra00478h base for sophisticated nanosynthesis such as nucleation of graphenes ${ }^{11}$ and stress-controlled processes. ${ }^{12,13}$

However, efficiency of deposition and formation of highquality hydrogenated amorphous silicon films, as well as the level of structural and morphological control of the ready heterojunctions still need significant improvement. An apparent lack of advanced techniques for highly controllable, environmentally friendly fabrication of the Si film-based devices calls for further progress in studying technological aspects and growth/nucleation mechanisms in particular. Importantly, modern methods such as plasma-based and ultrasonic techniques used for the fabrication of hydrogenated amorphous silicon films feature exceptionally complex nucleation and formation routes, and some key processes are still questionable. No significant breakthrough in quality control is possible without deep understanding and thorough look into the chemistry and physics of the amorphous silicon film formation.

Up to day, extensive experimental efforts were undertaken to reveal the effect of plasma-based process conditions ${ }^{\mathbf{1 4}}$ on the quality and structure of the deposited films. ${ }^{15,16}$ In particular, effect of plasma power density and incident precursor flux variation onto the film structure, morphology and characteristics has been investigated with the involvement of subsequent sophisticated film characterization. ${ }^{17,18}$ Effect of hydrogenation was studied experimentally with the aim to better control the structure and morphology of hydrogenated silicon. ${ }^{19}$ It was in 
particular demonstrated that hydrogen is able to modify the structure and properties of crystalline (c-Si), as well as amorphous (a-Si:H). ${ }^{20}$ The interaction of hydrogen plasma with c-Si surface gives rise to a large variety of phenomena ${ }^{21,22}$ and thus significantly complicates the picture.

Nevertheless, much deeper understanding of the processes and physics behind them is required to lunch the devices utilizing the hydrogenated amorphous silicon films to the orbit of wide commercial application. Complicity of the processes which involve a great variety of species negates the efficiency of experimental methods and invokes the detailed, sophisticated theoretical and numerical investigations capable of revealing unexplored details of the nucleation, growth and structure formation. Indeed, several approaches allowed revealing very important facts. Since silane $\left(\mathrm{SiH}_{4}\right)$ is used as the most common feedstock gas in the plasma-based processes, and as a result, the dominant species in the silane discharge are $\mathrm{SiH}_{3}, \mathrm{SiH}_{2}$, and $\mathrm{SiH}$ radicals, as well as ions of various degrees. It's difficult to clear the roles by experiment, and in particular, $\mathrm{SiH}_{3}$ radicals were supposed to be the key precursor for the film nucleation. Indeed, the kinetic Monte Carlo simulations have demonstrated that $\mathrm{SiH}_{3}$ radicals play an important role in the formation of microcrystals, ${ }^{23}$ while $\mathrm{SiH}_{2}$ is a precursor for dust particle formation ${ }^{24}$ and plasma-induced polymerization. ${ }^{25}$ It has been in particular found that at low power densities and low pressures, silyl radical $\left(\mathrm{SiH}_{3}\right)$ is the dominant deposition precursor leading to device-quality films. ${ }^{26}$

Despite the considerable experimental achievements, some key processes underlying formation of the hydrogenated amorphous silicon films still remain questionable. Apparently, detailed multiscale simulations are required to uncover the most complicated processes intrinsic to the film formation. The attempts of in-depth but still not comprehensive studies of the amorphous film growth are known. Models of a-Si:H film growth based on a thermodynamic approach have been reported. In a model developed by Bakos et al. only limited chemical reactions on surface were taken into account. ${ }^{27}$ As a result, the model was not able to describe how the film actually nucleate and grow. Other approaches to the modelling of a-Si:H growth proposed also did not ensure an adequate description of the film structure. ${ }^{28}$ Recently, Crose et al. developed a hybrid kinetic Monte Carlo (kMC) algorithm to estimate the interdependence of the gas phase and film growth phenomena by considering only four basic surface processes: physisorption, surface migration, hydrogen abstraction and chemisorption; however, other surface phenomena of key importance such as ion sputtering and direct chemisorption of radicals to the dangling bonds were ignored. ${ }^{29,30}$ Many experimental studies have meanwhile reported significant contribution of the incident ions on the thin film surface morphology and properties. ${ }^{31,32}$

Motivated by the above considerations, we used a sophisticated, multiscale model composed of several self-consisted submodels to simulate the plasma-substrate interaction, reveal the key growth processes and hence, to ensure much higher level of control over the film structure and properties. The global surface sticking model takes into account $\mathrm{SiH}_{3}$ and $\mathrm{H}$ radicals reacting in plasma and on the surface. The non-radical neutrals (i.e., $\mathrm{SiH}_{4}$ and $\mathrm{H}_{2}$ ) assumed reflected back to the discharge. The surface reaction and sticking probability of each radical are essentially material-specific, and the substrate temperature influences the surface reaction probabilities. Based on this model an extension of our understanding of the film nucleation and growth mechanisms is given on the basis of the detailed simulations and calculations.

The rest of paper is organized as follows. Section II describes in short the applied numerical method (the detailed model can be found in the ESI available $\dagger$ ), together with some basic underlying physics of Si:H film growth. In Section III, the numerical results modelling the contributions of different fluxes during the film growth are presented and discussed. Dependences of the film characteristics on the plasma and substrate parameters are also addressed. The plasma-sheath structure, incident ion and radical fluxes to the surface of growing films, and other control parameters are examined to optimize the Si film growth parameters. The paper is concluded with Section IV presenting a summary of the main results.

\section{Physics and numerical method}

\section{Structure of the model}

The whole model of nucleation and growth of hydrogenated amorphous silicon film comprises three relatively independent sub-models integrated into the complex multi-scale model capable of adequately describe the whole film formation process. In particular,

(i) Plasma sheath model describes the basic processes in the plasma-surface interface;

(ii) Ion/radical surface interaction model addresses the interaction of active species with surface; and

(iii) Growth model describes the film formation characteristics such as growth rate.

Fig. 1 illustrates the basic processes taken into account in the complex model. In addition to the processes in plasma bulk (ionization, radical formation etc.), the model accounts for a number of surface- and sheath-based processes such as adsorption and desorption of $\mathrm{SiH}_{3}, \mathrm{H}$ abstraction by $\mathrm{H}$ radical and $\mathrm{SiH}_{3}$ radical, $\mathrm{SiH}_{3}$ abstraction by $\mathrm{SiH}_{3}$ radical, direct chemisorption of $\mathrm{SiH}_{3}$ and $\mathrm{H}$ to the dangling bond, hopping of adsorbed $\mathrm{SiH}_{3}$ on hydride sites and chemisorption of adsorbed $\mathrm{SiH}_{3}$ to the dangling bond, and ion sputtering of hydrogen from surface.

During simulations we have assumed that the process is based on the inductively-coupled plasma (ICP) process, ${ }^{33}$ commonly used for the deposition of hydrogenated Si films. The typical process parameters used for simulations are listed in Table 1.

The first sub-model (plasma sheath model) uses a multi-fluid approach and incorporates the continuity, momentum, and Poisson's equations. It allows simulation of the density and velocity of charged particles in plasma-surface sheath. To determine the energy and flux of the positive ions and reactive radicals to the substrate surface, the sheath governing equations were numerically solved in one $(Z)$ dimension. 


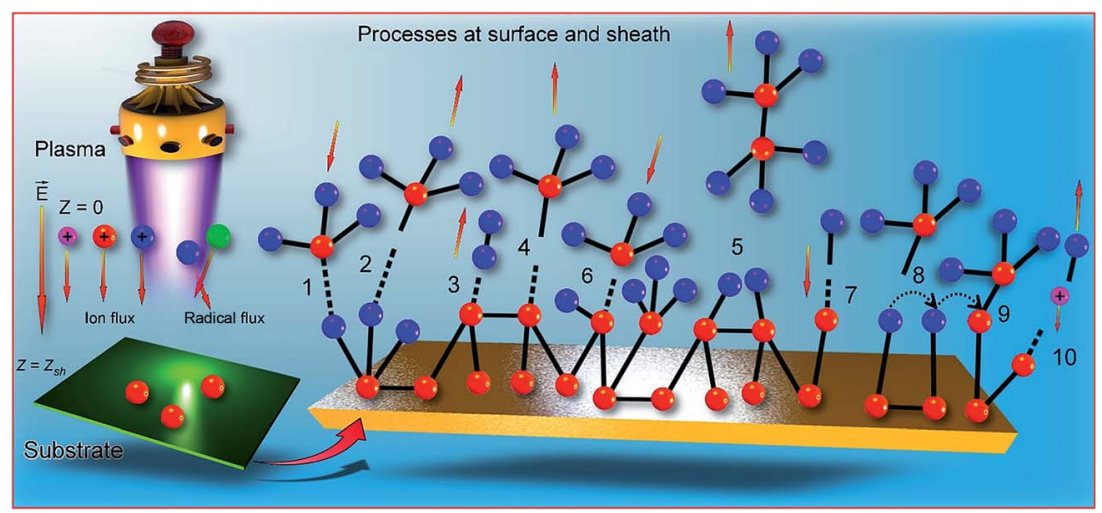

Fig. 1 Schematic representation of the basic surface reactions of $\mathrm{SiH}_{3}$ and $\mathrm{H}$ radicals during a-Si:H film growth, used in the model. Left panel: processes in plasma. Right panel: processes on surface and in the plasma-surface sheath: (1) adsorption of $\mathrm{SiH}_{3}$; (2) desorption of $\mathrm{SiH}_{3}$; (3) $\mathrm{H}$ abstraction by $\mathrm{H}$ radical; (4) $\mathrm{H}$ abstraction by $\mathrm{SiH}_{3}$ radical; (5) $\mathrm{SiH}_{3}$ abstraction by $\mathrm{SiH}_{3}$ radical; (6) direct chemisorption of $\mathrm{SiH}_{3}$ into dangling bond; (7) direct chemisorption of $\mathrm{H}$ into dangling bond; (8) hopping of adsorbed $\mathrm{SiH}_{3}$ on hydride sites; (9) chemisorption of adsorbed $\mathrm{SiH}_{3}$ into dangling bond; and (10) ion sputtering of mono-hydride sites.

Table 1 The typical process parameters used in the simulations

\begin{tabular}{|c|c|c|}
\hline Parameters & Notation & Value \\
\hline Electron temperature & $T_{\mathrm{e}}$ & $1-3 \mathrm{eV}$ \\
\hline Ion temperature & $T_{\mathrm{i}}=T_{\mathrm{g}}$ & $0.01-0.15 \mathrm{eV}$ \\
\hline Plasma density & $n_{\mathrm{e} 0}$ & $10^{10}$ to $10^{12} \mathrm{~cm}^{-3}$ \\
\hline Neutral gas pressure & $p_{0}$ & 20-100 mTorr \\
\hline Substrate potential & $V_{\mathrm{dc}}$ & 0 to $-300 \mathrm{~V}$ \\
\hline Substrate temperature & $T_{\mathrm{s}}$ & $300-700 \mathrm{~K}$ \\
\hline Percentage of $\mathrm{SiH}_{3}$ gas & $r_{\mathrm{SiH}_{3}}$ & $10-40 \%$ \\
\hline Percentage of $\mathrm{H}$ gas & $r_{\mathrm{H}}$ & $5-35 \%$ \\
\hline Percentage of Ar gas & $r_{\mathrm{Ar}}$ & $55-85 \%$ \\
\hline
\end{tabular}

The ion/radical surface interaction and growth sub-models describe the interaction of incoming species with the substrate surface and growing film. These models use rate equation, Eley-Rideal mechanism and surface diffusion formalism. The surface coverage of different species is expressed in terms of a set of coupled non-linear timedependent differential equations that accounts for the generation and consumption of these sites. If we assume a steady state growth, these differential equations reduce to a set of non-linear algebraic equations. The steady-state site balances for $\theta_{\mathrm{db}}, \theta_{\mathrm{SiH}}$ and $\theta_{\mathrm{SiH}_{3}}$ are obtained in the next steps.

More details can be found in the ESI. $\dagger$

Table 2 lists the main chemical reactions and the relevant specific barrier energies (probabilities) for the major values involved in all three sub-models. The detailed description of all sub-models, processes, and mathematical formulations can be found in the ESI. $\dagger$

\section{Results and discussion}

\section{Conditions of simulations}

This section presents the results of numerical simulations obtained using multiscale model introduced in the previous section. A low-temperature, low-pressure plasma ignited and sustained in $\mathrm{Ar} / \mathrm{H}_{2} / \mathrm{SiH}_{4}$ gas mixtures was examined. First, the contribution of individual fluxes in the growth kinetic of each surface process has been studied. Then, the dependencies of the film growth characteristics on plasma and substrate parameters was explored.

To study the effect of each discharge parameter on the characteristics of Si film, we have assumed that the other values are kept constant. The initial plasma and surface parameters in the numerical calculations have been selected on the base of experiments conducted in high-density inductively coupled plasmas. ${ }^{34,35}$ In a typical ICP discharge the $\mathrm{SiH}_{4}$ molecules break down, and the neutral species and ions hit the substrate at the room temperature $(300-400 \mathrm{~K}){ }^{36}$ hence, this justifies the low substrate temperature assumption used in the numerical simulations. The range of variation for different parameters used in the simulation is given in Table 1.

\section{Effect of dangling bonds}

The surface coverages of dangling bonds, $\mathrm{SiH}_{3}$ and $\mathrm{H}$ monohydride radicals, film growth rate, as well as the hydrogen content are plotted for different substrate temperatures in Fig. 2. From these graphs one can see that the deposition rate increases quite slowly at temperature below $450 \mathrm{~K}$, but then increases quicker as temperature rises above $450 \mathrm{~K}$. The fraction of dangling bond sites also shows non-monotonic behaviour with increasing surface temperature $T_{\mathrm{s}}$ : it first decreases with increasing $T_{\mathrm{s}}$ up to $450 \mathrm{~K}$, and then increases at higher temperatures.

The steady-state fraction of surface dangling bonds is determined by the balance between their generation and annihilation rates. Under low-substrate-temperature condition (300 $\mathrm{K}<T_{\mathrm{s}}<450 \mathrm{~K}$ ), surface dangling bonds are mostly produced by ion sputtering rather than $\mathrm{H}$ abstraction reactions involving $\mathrm{SiH}_{3}$ and $\mathrm{H}$ radicals, since the abstraction reaction shows slower rate at lower temperatures. On the other hand, surface dangling bonds are saturated due to surface diffusion and subsequent chemisorption of 
Table 2 The main chemical reactions, as well as barrier energies and probabilities for the major values involved in all three sub-models

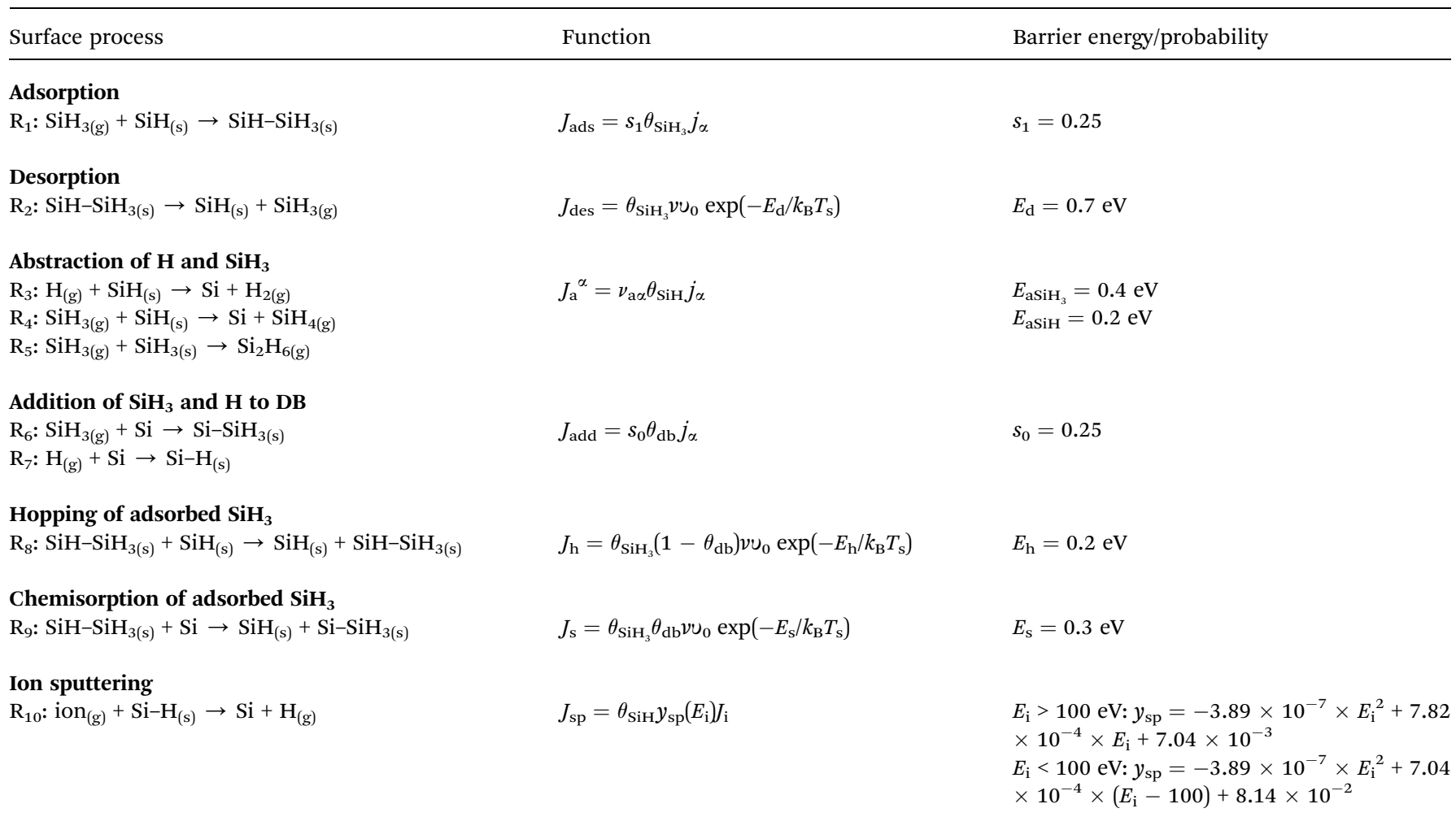

physisorbed $\mathrm{SiH}_{3}$, as well as direct chemisorption of $\mathrm{SiH}_{3}$ radicals; importantly, these processes are almost independent of substrate temperature.

The number density of surface dangling bonds under the steady-state conditions remarkably reduces with increasing
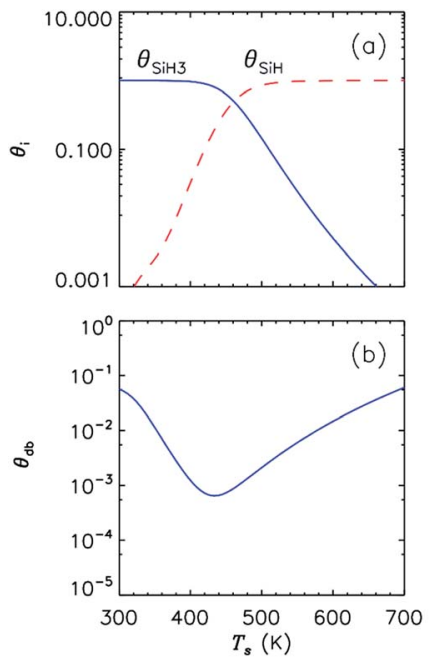
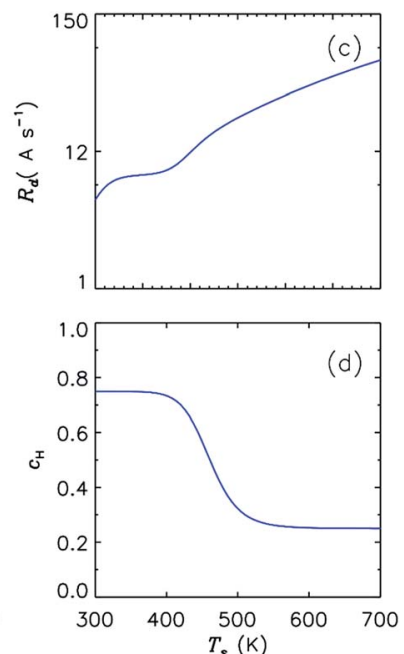

Fig. 2 Surface coverage of $\mathrm{SiH}$ and $\mathrm{SiH}_{3}$ sites (a), surface coverage of dangling bonds (b), growth rate (c), and hydrogen content (d) as functions of substrate temperature. Other parameters are the same as in Table 1. The deposition rate increases quite slowly at temperature below $450 \mathrm{~K}$, but then increases quicker as temperature rises above $450 \mathrm{~K}$. The fraction of dangling bond sites also shows non-monotonic behaviour. substrate temperature due to slow $\mathrm{H}$ abstraction reaction and thermally enhanced surface diffusion of $\mathrm{SiH}_{3}$ radicals, thus resulting in minimum defect density at substrate temperature of $450 \mathrm{~K}$. On the other hand, the increase in the density of dangling bond sites at substrate temperature exceeding $450 \mathrm{~K}$ could be explained by the increase in the rate of thermal abstraction of $\mathrm{H}$ covering the substrate surface. As can be also seen in Fig. 2(d), the hydrogen content in the growing film decreases with increasing of $T_{\mathrm{S}}$ due to the enhancement of thermal $\mathrm{H}$ abstraction; this is quite compatible with experimental findings. ${ }^{37}$

\section{Growth rate}

Investigation of the growth rate dependencies also enables us to find the most significant growth process at different temperatures. Eqn (16) (see ESI available $\dagger$ ) includes the two distinct mechanisms for silyl incorporation into the growing film: (i) direct chemisorption to the dangling bonds; and (ii) radical physisorption, diffusion, and chemisorption to the dangling bonds. The contribution of each of these individual terms is plotted as a function of substrate temperature in Fig. 3.

Specifically, we have found that the chemisorption of physisorbed silyl radicals to the dangling bond site $\left(R_{\mathrm{s}}\right)$ dominates over direct chemisorption to dangling bonds $\left(R_{\text {add }}\right)$ at substrate temperature $T_{\mathrm{s}}$ of $350 \mathrm{~K}$, while $R_{\text {add }}$ becomes the dominant process at lower temperature. Actually, the chemisorption flux of physisorbed radicals is determined by the temperature- 


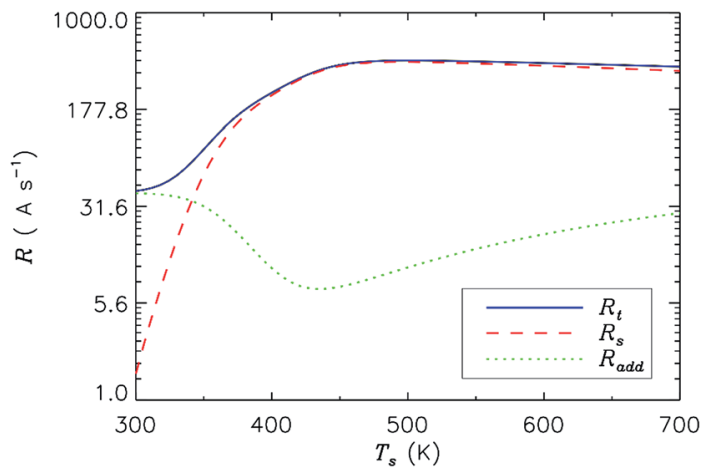

Fig. 3 Relative contributions of different pathways towards film growth under a typical deposition rate conditions. Other parameters are the same as in Table $1 . R_{\mathrm{s}}$, chemisorption of physisorbed silyl radical to a dangling bond site; $R_{\text {add, }}$, direct chemisorption into dangling bonds.

dependent surface coverage of dangling bonds, rate of chemisorption to dangling bonds, and surface coverage of physisorbed radicals. The deposition rate shows weak temperature dependence at $T_{\mathrm{s}} \approx 350 \mathrm{~K}$ because the physisorbed radical surface coverage decreases with $T_{\mathrm{s}}$, while the rate of physisorbed radical hopping and chemisorption increases; at $T_{\mathrm{s}} \approx$ $450 \mathrm{~K}$, both chemisorption and addition fluxes lead to the increasing of substrate temperature, thus opening important possibility for the structure control. ${ }^{38,39}$ Owing to the $\mathrm{H}$ abstraction reactions which strongly dominate in PECVD deposition of a-Si:H films, ions can play an important role in the creation of surface dangling bonds.

\section{Effect of ion sputtering}

Fig. 4 illustrates the effect of ion sputtering on temperature dependence of the addition flux $\left(J_{\text {add }}\right)$ and chemisorption flux of adsorbed $\mathrm{SiH}_{3}$ to the dangling bonds $\left(J_{\mathrm{s}}\right)$, growth rate, and surface coverage of dangling bonds. From this graphs it is seen that the ion sputtering dominates at low substrate temperatures $\left(T_{\mathrm{s}} \approx 450 \mathrm{~K}\right)$, thus leading to enhanced surface density of the dangling bonds.

These graphs show that the flux of physisorbed silyl radicals, their direct chemisorption and consequently the growth rate increase with the substrate temperature. According to eqn (16) shown in the ESI, $\uparrow$ the $\mathrm{H}$ abstraction process in particular is responsible for the creation of dangling bonds in the absence of ion sputtering, while the chemisorption leads to the consumption of dangling bond. Then, when substrate temperature is below $450 \mathrm{~K}$, chemisorption overcomes the $\mathrm{H}$ abstraction and hence, lower density of dangling bond sites is established. The picture changes significantly in the presence of ion sputtering: temperature distribution of dangling bond coverage shows a minimum at around $450 \mathrm{~K}$, as one can see in Fig. 4. These results are in good agreement with the relevant experimental results. ${ }^{40,41}$ The hydrogen abstraction increases with the surface temperature $T_{\mathrm{s}}$, this in turn leads to the increase of dangling bond density and growth rate of the film.

Fig. 5 shows the dependencies of dangling bond coverage and film growth rate on substrate temperature for the three
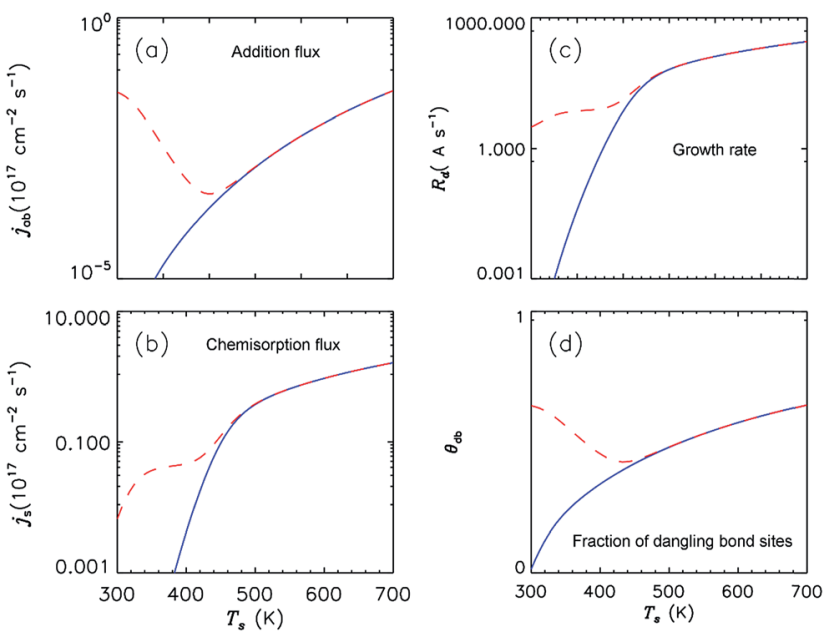

Fig. 4 Dependence of addition flux $\left(J_{\text {add }}\right)$, chemisorption flux of physisorbed radicals $\left(J_{s}\right)$, growth rate $\left(R_{d}\right)$, and fraction of dangling bond site $\left(\theta_{\mathrm{db}}\right)$ on substrate temperature. The solid curves correspond to the case of the absence of ion sputtering, the dashed curves correspond to the cases in which the ion sputtering effect is taken into account. Other parameters are the same as in Table 1.

different values of negative bias $\left(V_{\mathrm{dc}}\right)$ applied to the substrate surface. Apparently, increase of $V_{\mathrm{dc}}$ and consequently the ion energy and flux result in the increase of rates of dangling bond generation and film growth. As seen from Fig. 5, the effect of substrate bias is more pronounced at lower substrate temperatures, while the effects of other dangling bond production processes is less important, in good agreement with the relevant experimental results. ${ }^{34}$
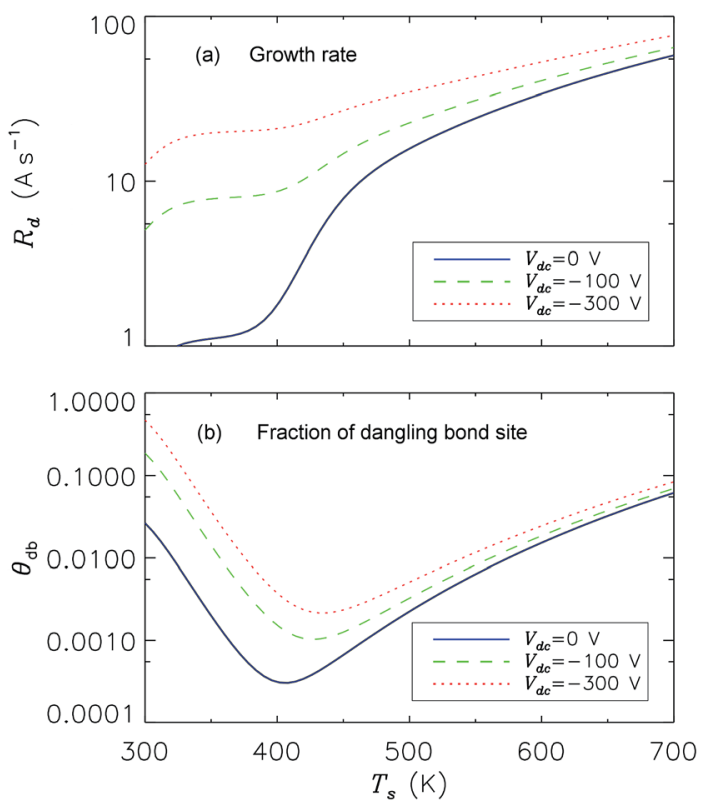

Fig. 5 Dependence of (a) growth rate and (b) fraction of dangling bond site on the substrate temperatures with the substrate bias as a parameter. Other parameters are the same as in Table 1. 


\section{Effect of ion substrate temperature, pressure and electric} bias

To explore the impacts of substrate temperature and applied bias on the surface coverage of dangling bonds and film growth parameters, effects of the individual fluxes participating in the surface processes was examined. Fig. S1 (ESI $\dagger$ ) presents the ion sputtering flux $\left(J_{\mathrm{sp}}\right)$, flux of radicals to dangling bond sites $\left(j_{\mathrm{sp}}\right)$, chemisorption flux of adsorbed radicals to dangling bonds $\left(J_{\mathrm{s}}\right)$, radical adsorption $\left(j_{\text {ad }}\right)$ and hydrogen abstraction fluxes $\left(j_{\mathrm{ab}}\right)$ for different applied biases and substrate temperatures. Since the sputtering flux is directly proportional to the flux of incident ions, the enhancement of $j_{\mathrm{sp}}$ with the substrate bias $V_{\mathrm{dc}}$ is same for all substrate temperatures. On the other hand, the rates of other surface processes show an exponential dependence on substrate temperature, and hence, the bias effect becomes less significant at higher values of $T_{\mathrm{s}}$.

Working gas pressure $\left(p_{0}\right)$ is one of the most important parameters used to control the rates of generating various radicals and ions hence, it ensures efficient control of the film growth rate. Fig. 6 presents the effects of working gas pressure on of the film growth parameters at various surface temperatures. Importantly, the growth rate shows a non-monotonic dependence on the gas pressure. At relatively low values of surface temperature (about $450 \mathrm{~K}$ ), the film growth rate decreases with the gas pressure increasing, while at higher temperatures, the film growth rate always increases with the gas pressure.

It is noteworthy that the incident flux of ions is almost independent on the total gas pressure, while the flux of neutral radicals increases with gas pressure, and kinetic energy of the incident ions decreases with gas pressure. ${ }^{42}$ The production rates of both $\mathrm{H}$ and $\mathrm{SiH}_{3}$ radicals at higher pressure are enhanced by the electron impact reactions. Consequently, the consumption rate of dangling bonds increases at low substrate temperature due to the enhanced radical addition fluxes. At higher temperature, the increase of gas pressure is causes an
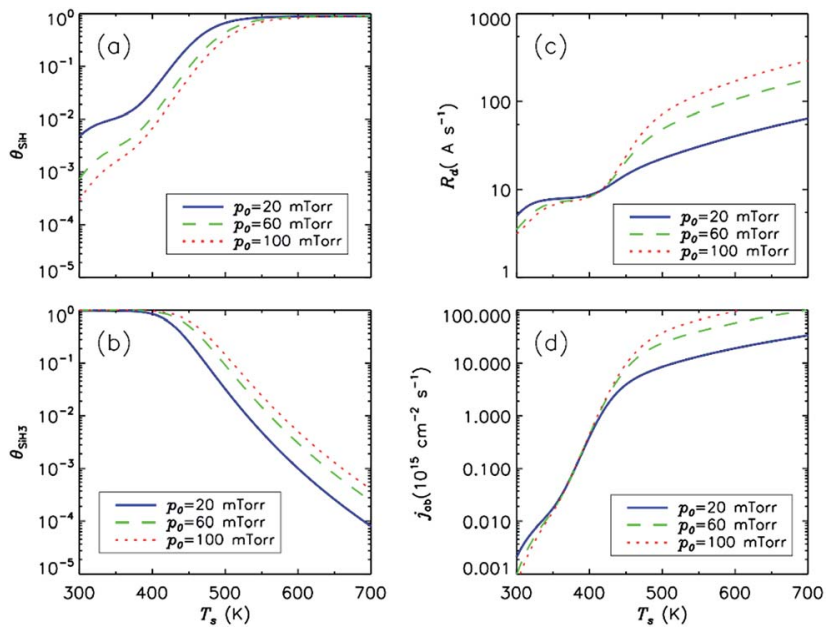

Fig. 6 Surface coverages of $\mathrm{SiH}_{3}, \mathrm{H}$, and $\mathrm{DB}$, and the film growth rate as functions of substrate temperature for three different pressures. Other parameters are the same as Table 1. increase in the hydrogen abstraction rate and as a result, it leads to an enhancement of the dangling bond production and film growth rates.

\section{Effect of hydrogen}

To study the effect of $\mathrm{H}$ radical concentration in the plasma sheath on the film growth rate, dangling bond coverage and growth rates, we have plotted these parameters in Fig. S2 (ESI available $\dagger$ ) as functions of surface temperature for different percentage of hydrogen $r_{\mathrm{H}}$. It was found that both the dangling bond coverage and growth rate show a non-monotonic dependence on $r_{\mathrm{H}}$. At low substrate temperatures, coverage and growth rate decrease with $r_{\mathrm{H}}$, but invert behaviour is seen at higher temperatures. We suppose here that the initial decrease is due to dominance of the $\mathrm{H}$ radical addition over abstraction for $T_{\mathrm{s}} \approx 450 \mathrm{~K}$; on the other hand, significant $\mathrm{H}$ abstraction at higher temperatures leads to the increase of dangling bond density and hence, to the increase of film growth rate with $r_{\mathrm{H}}$.

\section{Summary and conclusions}

Plasma-surface processes play a significant role in the nucleation, growth, and structure formation of thin films and structures including nanoscaled a-Si: $\mathrm{H}$ interfaces. Experimental techniques ensure methodical investigation of integral dependencies and characteristic, while the role of specific physical and chemical processes (such as, e.g., effect of certain radicals etc.) cannot be revealed by the application of direct experimental methods.

However, such dependencies are critically important for designing and discovering novel material with important properties.

Here we report on the novel, important results obtained by the use of a sophisticated multiscale model based on selfconsistent surface and plasma kinetics sub-models, including one-dimensional plasma sheath approximation. Specifically, this model was developed and used to study the details of the deposition and structure formation of a-Si:H films from reactive low temperature plasma environment containing silane, hydrogen, and argon gas mixture.

The model considers the whole range of the key and important processes such as generation, adsorption and desorption of $\mathrm{SiH}_{3}$ radicals on the film surface, hydrogen abstraction by incoming $\mathrm{SiH}_{3}$ and $\mathrm{H}$ radicals from plasma sheath, attachment of incoming $\mathrm{SiH}_{3}$ and $\mathrm{H}$ radicals to the dangling bonds, hopping of adsorbed $\mathrm{SiH}_{3}$ on the hydride sites, ion sputtering of $\mathrm{H}$ atoms from the film, and chemisorption of adsorbed $\mathrm{SiH}_{3}$ on the dangling bond sites. The steady-state surface site balance equations were solved to obtain the surface coverage of dangling bonds and each radical species. The effects of surface temperature, ion flux, energy and other plasma-sheath parameters were examined in details.

It was found that at low substrate temperatures and within the reasonable range of ion flux, the surface is primarily covered by $\mathrm{SiH}_{3}$ radicals. The dominance of $\mathrm{SiH}_{3}$ on the surface at low temperatures is accompanied by a relatively slow hydrogen 
attachment rate, which supports the hypothesis that $\mathrm{SiH}_{3}$ is the main precursor for a-Si:H deposition. At higher temperatures, the hydrogen abstraction proceeds via hydrogen attachment to $\mathrm{SiH}_{3}$ radicals, resulting in fewer higher hydrides on the surface and higher rate of dangling bond production. The abstraction reactions can be accelerated by increasing applied bias voltage and consequently by enhancement of the ion flux which generates more dangling bonds on the surface at low substrate temperatures.

We have also found that the fraction of dangling bonds sites exhibits the non-monotonic behaviour with increasing surface temperature, which was explained in terms of combination of hydrogen with $\mathrm{SiH}_{3}$ diffusing on surface, and domination of chemisorption over the chemisorption of the physisorbed radicals at low temperatures.

Hence, the key role of hydrogen is confirmed and moreover, key processes were identified and route for the efficient, technique-enabled control are specified.

The presented results were compared with the experimental data and have demonstrated a good agreement with the experimental findings obtained for the deposition of amorphous hydrogenated films.

\section{Acknowledgements}

This work was partially supported by the National Research Foundation (EDB/OSTIn); Academic Research Fund (AcRF) Tier 1, Singapore; the Ministry of Science, Research and Technology of Iran; Iran Nanotechnology Initiative Council; CSIRO CEO Science Leadership program; and the Australian Research Council. I. L. acknowledges support from the School of Chemistry, Physics and Mechanical Engineering, Science and Engineering Faculty, Queensland University of Technology.

\section{References}

1 J. Dong and B. Lin, RSC Adv., 2016, 6, 110409-110415.

2 C. Tang, Z. Yan, Q. Wang, J. Chen, M. Zhu, B. Liu, F. Liu and C. Sui, $R S C$ Adv. , 2015, 5, 81866-81874.

3 G. Das, S. Mandal, S. Dhar, S. Bose, S. Mukhopadhyay, C. Banerjee and A. K. Barua, J. Mater. Sci.: Mater. Electron., 2017, 28, 5746-5753.

4 C. Shin, J. Park, J. Jung, S. Bong, S. Kim, Y.-J. Lee and J. Yi, Mater. Res. Bull., 2014, 60, 895.

5 M.-K. Kang, S. J. Kim and H. J. Kim, Sci. Rep., 2014, 4, 6858.

6 T. K. Subramanyam, V. Nagendra, P. Goutham, P. Kumar and K. N. Subramanya, Microelectron. Solid State Electron., 2016, 5, 14-18.

7 M. A. Marrs and G. B. Raupp, Sensors, 2016, 16, 1162, DOI: 10.3390/s16081162.

8 R. He, et al., Adv. Mater., 2016, 28, 5939-5942.

9 E. Markevich, G. Salitra, A. Rosenman, Y. Talyosef, D. Aurbach and A. Garsuch, RSC Adv., 2014, 4, 48572-48575. 10 M. Li and Y. Yue, RSC Adv., 2014, 4, 23010-23016.

11 I. Levchenko, K. Ostrikov, J. Zheng, X. Li, M. Keidar and K. B. K. Teo, Nanoscale, 2016, 8, 10511-10527.
12 E. Johlin, N. Tabet, S. Castro-Galnares, A. Abdallah, M. I. Bertoni, T. Asafa, J. C. Grossman, S. Said and T. Buonassisi, Phys. Rev. B: Condens. Matter Mater. Phys., 2012, 85, 075202.

13 I. Levchenko, U. Cvelbar and K. Ostrikov, Appl. Phys. Lett., 2009, 95, 021502.

14 M. Hundt, P. Sadler, I. Levchenko, M. Wolter, H. Kersten and K. Ostrikov, J. Appl. Phys., 2011, 109, 123305.

15 J. W. M. Lim, C. S. Chan, L. Xu, T. M. Ong, S. Y. Huang, D. Y. Wei, Y. N. Guo and S. Xu, Procedia Eng., 2016, 139, 56-63.

16 W. Guo, L. Zhang, J. Bao, F. Meng, J. Liu, D. Wang, J. Bian, W. Liu, Z. Feng, P. J. Verlinden and Z. Liu, J. Appl. Phys., 2015, 54, 041402.

17 Y. Rong-Hwei, Y. Tai-Rong, C. Te-Cheng, L. Shih-Yung and H. Jyh-Wong, IEEE Trans. Electron Devices, 2008, 55, 978-985.

18 J. Fang, I. Aharonovich, I. Levchenko, K. Ostrikov, P. G. Spizzirri, S. Rubanov and S. Prawer, Cryst. Growth Des., 2012, 12, 2917-2922.

19 S. Sriraman, S. Agarwal, E. S. Aydil and D. Maroudas, Nature, 2002, 418, 62-65, DOI: 10.1038/nature00866.

20 M. Plaza, I. G. Hemme, E. G. Hernansanz, R. G. Díaz, G. O. Ariza and J. P. Millán, Proc. Spanish Conference on Electron Devices, IEEE, 2013, pp. 337-340, ISBN 978-1-46734666-5.

21 R. Amrani, P. Abboud, L. Chahed and Y. Cuminal, Cryst. Struct. Theory Appl., 2012, 1, 62-67.

22 S. Q. Xiao and S. Xu, J. Phys. D: Appl. Phys., 2011, 44, 174033.

23 S. C. Pandey, T. Singh and D. Maroudas, J. Chem. Phys., 2009, 131, 034503.

24 M. Wolter, I. Levchenko, H. Kersten and K. Ostrikov, Appl. Phys. Lett., 2010, 96, 133105.

25 M. Takai, T. Nishimoto, M. Kondo and A. Matsuda, Sci. Technol. Adv. Mater., 2001, 2, 495-503.

26 S. Xiao, S. Xu and K. Ostrikov, Mater. Sci. Eng., R, 2014, 78, 1.

27 T. Bakos, M. S. Valipa and D. Maroudas, J. Chem. Phys., 2007, 126, 114704.

28 Z. Mrázková, K. Postava, A. Torres-Rios, M. Foldyna, P. Roca i Cabarrocas and J. Pištora, Procedia Mater. Sci., 2016, 12, 30135.

29 M. Crose, J. Sang-Il Kwon, M. Nayhouse, D. Ni and P. D. Christofides, Chem. Eng. Sci., 2015, 136, 50-61.

30 M. Crose, J. Sang-Il Kwon, M. Nayhouse, D. Ni and P. D. Christofides, Renewable Energy, 2017, 100, 129-140.

31 S. Lebib and P. Roca i Cabarrocas, J. Appl. Phys., 2005, 97, 104334.

32 C. Wen, H. Xu, H. Liu, Z. Li and W. Shen, Nanotechnology, 2013, 24, 455602.

33 I. Levchenko, M. Keidar, S. Xu, H. Kersten and K. Ostrikov, J. Vac. Sci. Technol., B: Nanotechnol. Microelectron.: Mater., Process., Meas., Phenom., 2013, 31, 050801.

34 M. Hishida, T. Sekimoto, M. Matsumoto and A. Terakawa, Energies, 2016, 9, 42.

35 J. Ge, M. Tang, J. Wong, Z. Zhang, T. Dippell, M. Doerr, O. Hohn, M. Huber, P. Wohlfart, A. G. Aberle and T. Mueller, Int. J. Photoenergy, 2014, 752967. 
36 D. Y. Wei, S. Q. Xiao, S. Y. Huang, C. S. Chan, H. P. Zhou, L. X. Xu, Y. N. Guo, J. W. Chai, S. J. Wang and S. Xu, J. Phys. D: Appl. Phys., 2013, 46, 215501.

37 B. Korevaar, G. Adriaenssens, A. Smets, W. Kessels, H. Z. Song, M. van de Sanden and D. Schram, J. Non-Cryst. Solids, 2000, 266-269, 380-384.

38 J. Huran, P. Boháček, V. N. Shvetsov, A. P. Kobzev, A. Kleinová, V. Sasinková, N. I. Balalykin, M. Sekáčová and J. Arbet, 21st Int. Symposium on Plasma Chem., ISPC 21, Cairns, Queensland, Australia, 2013.
39 S. Askari, I. Levchenko, K. Ostrikov, P. Maguire and D. Mariotti, Appl. Phys. Lett., 2014, 104, 163103.

40 D. Marra, W. Kessels, M. van de Sanden, K. Kashefizadeh and E. Aydil, Surf. Sci., 2003, 530, 1.

41 Z. Marvi, S. Xu, G. Foroutan and K. Ostrikov, Phys. Plasmas, 2015, 22, 013504.

42 I. Levchenko, M. Romanov, O. Baranov and M. Keidar, Vacuum, 2003, 72, 335-344. 